\title{
Phase II/III Study of Lisdexamfetamine Dimesylate in Japanese Pediatric Patients with Attention-Deficit/Hyperactivity Disorder
}

\author{
Hironobu Ichikawa, MD, PhD, ${ }^{1}$ Tasuku Miyajima, MD, PhD, ${ }^{2}$ Yushiro Yamashita, MD, PhD, ${ }^{3}$ \\ Masakazu Fujiwara, $\mathrm{PhD},{ }^{4}$ Akimasa Fukushi, $\mathrm{MSc}^{5}$ and Kazuhiko Saito, MD, $\mathrm{PhD}^{6}$
}

\begin{abstract}
Objective: To further define the efficacy and safety profiles of lisdexamfetamine dimesylate (LDX) in Japanese pediatric patients with attention-deficit/hyperactivity disorder (ADHD).

Methods: This was a multicenter, randomized, double-blind, placebo-controlled study of LDX 30, 50, or 70 mg/day for 4 weeks in 76 patients 6-17 years of age with ADHD in Japan. The primary efficacy endpoint was the change in the ADHD Rating Scale-IV (ADHD-RS-IV) total score from baseline to 4 weeks. Secondary efficacy endpoints were: Conners' Third Edition (Japanese version) Parent Rating Scale (Conners 3), Clinical Global Impression-Improvement (CGI-I) scale, and Parent Global Assessment (PGA) scale.

Results: Change in the ADHD-RS-IV total score from baseline to 4 weeks was significantly greater $(p<0.0001)$ in all LDX dosage groups versus placebo ( $30 \mathrm{mg},-16.38 ; 50 \mathrm{mg},-18.10 ; 70 \mathrm{mg},-16.47$; placebo, -2.78 ). At all time points, improvements (decreases) in the ADHD-RS-IV total score were significantly greater in all LDX groups versus placebo. At weeks 3 and 4, improvements from baseline in Conners 3 inattention plus hyperactivity/impulsivity subscale scores were significantly greater ( $p \leq 0.0082)$ for all LDX dosages versus placebo. At week 4, the proportion of LDX-treated patients "much improved" or "very much improved" was $61 \%-71 \%$ on the CGI-I scale $(p \leq 0.0019)$ and $56 \%-65 \%$ on the PGA scale $(p \leq 0.0170)$. LDX was generally well tolerated. The most frequent treatment-emergent adverse events (AEs) were decreased appetite, headache, and initial insomnia. No severe/serious AEs occurred, and no AEs specific to Japanese patients were evident.

Conclusions: The superiority of LDX 30, 50, and $70 \mathrm{mg}$ /day over placebo was confirmed in Japanese pediatric patients with ADHD, and no major safety or tolerability concerns were identified.
\end{abstract}

Keywords: adolescents, attention-deficit/hyperactivity disorder, children, efficacy, Japanese patients, lisdexamfetamine dimesylate, safety

\section{Introduction}

A TTENTION-DEFICIT/HYPERACTIVITY DISORDER (ADHD) is one of the most frequently diagnosed neurodevelopmental disorders in children and adolescents (Steer et al. 2012; Catala-Lopez et al. 2017), estimated to affect $\sim 5 \%-7 \%$ of school-aged children worldwide (Zimovetz et al. 2016; Catala-Lopez et al. 2017; Cortese et al. 2017; Taylor 2017). The condition is characterized by an age- inappropriate pattern of inattention and/or hyperactivity/impulsivity, accompanied by functional impairment (Clavenna and Bonati 2017; Cortese et al. 2017; Ruiz-Goikoetxea et al. 2017). Moreover, ADHD is significantly linked with an increased likelihood of unintentional injury (Ruiz-Goikoetxea et al. 2017), and is frequently associated with various comorbid psychiatric disorders, including anxiety and mood disorders, autism spectrum disorder, conduct disorder (CD), oppositional defiant disorder (ODD), sleep

\footnotetext{
${ }^{1}$ Japan Developmental Disorders Network, Tokyo, Japan.

${ }^{2}$ Department of Education for Childcare, Tokyo Kasei University, Saitama, Japan.

${ }^{3}$ Department of Pediatrics and Child Health, Kurume University School of Medicine, Fukuoka, Japan.

${ }^{4}$ Biostatistics Center, Shionogi \& Co., Ltd, Osaka, Japan.

${ }^{5}$ Clinical Research Department, Shionogi \& Co., Ltd, Osaka, Japan.

${ }^{6}$ Aiiku Counseling Office, Aiiku Research Institute, Imperial Gift Foundation Boshi-Aiiku-Kai, Tokyo, Japan.

Clinical Trial Registry: This clinical trial is registered at the Japan Pharmaceutical Information Center (Japic CTI-152770).

Funding: The study was funded by Shionogi \& Co. Ltd, and Shire Development LLC.

(C) Hironobu Ichikawa et al. 2019; Published by Mary Ann Liebert, Inc. This Open Access article is distributed under the terms of the Creative Commons Attribution Noncommercial License (http://creativecommons.org/licenses/by-nc/4.0/) which permits any noncommercial use, distribution, and reproduction in any medium, provided the original author(s) and the source are cited.
} 
disturbances, specific learning disorders, and substance use disorder (Cortese et al. 2017).

In Japan, a large-scale survey of elementary and middle school students attending universal classes revealed that about $3 \%$ of respondents had major problems with inattentiveness or hyperactivity/impulsivity (Japanese Ministry of Education, Culture, Sports, Science, and Technology 2012). An epidemiological survey of parents of more than 1000 children (4-12 years of age) living in the greater Tokyo area also identified an estimated ADHD prevalence of 7.7\% (Yamashita 2005). Thus, the overall burden posed by ADHD is enormous to patients-particularly regarding educational and vocational outcomes - and also to parents, caregivers, health care payors, and society in general (Steer et al. 2012; Zimovetz et al. 2016; Cortese et al. 2017).

The causes of ADHD are multifactorial and have not been definitively determined; the condition is also associated with nonspecific findings on neuroimaging (Tandon and Pergjika 2017). Data suggest that neurotransmitter deficits, genetic variation, environmental factors, and perinatal complications may all contribute to the multifaceted pathophysiology of ADHD. Much research has focused on ADHD treatments that promote release or potentiate the effects of neurotransmitters (e.g., dopamine, noradrenaline, or their precursors).

Traditionally, pharmacotherapy of ADHD has comprised various rapid-acting or sustained-release formulations of central nervous system (CNS) stimulants (e.g., amphetamine, methylphenidate) or nonstimulants (e.g., atomoxetine, clonidine, guanfacine) (Zimovetz et al. 2016; Cortese et al. 2017). Currently, in Japan, only three preparations are licensed for the treatment of ADHD: osmoticrelease oral system (OROS) methylphenidate, atomoxetine, and extended-release guanfacine. Thus, the number of pharmacotherapeutic options available for ADHD is limited, and more options are required to improve the management of the disorder.

Lisdexamfetamine dimesylate (LDX) is a pharmacologically inactive prodrug which, after oral absorption, is hydrolyzed in the bloodstream to dexamphetamine, a pharmacologically active form, and L-lysine (Steer et al. 2012; Hutson et al. 2014; Zimovetz et al. 2016; Adler et al. 2017; Dolder et al. 2017). This rate-limiting hydrolysis is mediated through peptidases associated with red blood cells (Heal et al. 2013). Relative to equimolar doses of dexamphetamine, maximum blood and CNS concentrations of LDX-derived dexamphetamine are attained later (Hutson et al. 2014; Dolder et al. 2017). That is, conversion of LDX to dexamphetamine is gradual. In addition, LDX has a reportedly prolonged clinical effect of up to 13 hours, and these factors may lead to slower CNS dopamine release, less euphoric activity, and lower potential for drug misuse (Jasinski and Krishnan 2009; Steer et al. 2012; Heal et al. 2013; Coghill et al. 2014; Adler et al. 2017; Dolder et al. 2017). Although the precise mechanism of dexamphetamine action in ADHD is not fully understood, the compound binds to plasma membrane monoamine transporters and inhibits monoamine (e.g., dopamine and noradrenaline) reuptake into presynaptic neurons in the prefrontal cortex. In addition, amphetamine enters presynaptic terminals and causes redistribution of monoamines from synaptic vesicles to the cytosol; the consequences are increased monoamine release from the cytosol through reverse transport at the membrane transporters, and increased monoamine levels in synapses (Sulzer et al. 2005).

LDX has been widely investigated and has demonstrated efficacy in several large-scale studies in children, adolescents, and adults with ADHD (Biederman et al. 2007; Findling et al. 2011; Steer et al. 2012; Stuhec et al. 2015; Zimovetz et al. 2016). It is currently marketed in various regions worldwide. An early phase I study conducted in the
United States showed that, for LDX doses of 20,50 , and $70 \mathrm{mg}$, no differences existed in the safety, tolerability, and pharmacokinetic profiles of LDX between Japanese and Caucasian individuals (data on file, Shionogi). Subsequently, an open-label phase II trial of LDX $30-70 \mathrm{mg}$ once daily for 4 weeks was conducted in Japan. In children 6-17 years of age with ADHD, a statistically significant decrease in the ADHD Rating Scale-IV (ADHD-RS-IV) total score from baseline was evident as early as 1 week after starting treatment with LDX $30 \mathrm{mg} /$ day (data on file, Shionogi). No severe or serious adverse events (AEs) occurred, and the overall profile of AEs was similar to that noted in studies of LDX conducted outside Japan. The abovementioned studies of LDX provided the basis and rationale for the current trial, which aimed to further define the efficacy and safety profiles of LDX in Japanese pediatric patients with ADHD.

\section{Methods}

This was a multicenter, placebo-controlled, randomized, double-blind, fixed-dose, comparative phase II/III study conducted at 23 sites in Japan in compliance with the Ministerial Ordinance on Good Clinical Practice (GCP) of the Pharmaceutical Affairs Law of Japan. The study protocol was approved by each center's Institutional Review Board. After a thorough description of the study, written informed consent to participate in the trial was obtained from the parents or legal guardian of all eligible patients. For patients $\geq 13$ years of age, written informed assent was obtained from the patient.

\section{Study participants}

Study participants were male or female children or adolescents (6-17 years of age) who met criteria as defined in the Diagnostic and Statistical Manual of Mental Disorders (DSM), 5th edition, for the principal diagnosis of ADHD (American Psychiatric Association 2013). At baseline, all patients had to have an ADHD-RS-IV total score $\geq 28$ to be eligible for randomization into the trial. Patients were to be functioning at an age-appropriate intellectual level, able to swallow capsules, and have thyroid-stimulating hormone and free thyroxine levels within normal ranges. Principal exclusion criteria included: serious disorders of the blood or bone marrow, heart, kidneys, liver, lungs; psychiatric comorbidity (e.g., bipolar disorder, schizophrenia); CD (excluding ODD); current tics; history of seizures; low or high bodyweight; hypertension; QTc interval (Fridericia adjusted; QTcF) >430 mseconds; substance use disorder; and pregnancy or lactation.

\section{Study objectives}

The primary study objective was to evaluate LDX efficacy in Japanese pediatric patients with ADHD and to confirm the superiority relative to placebo of LDX dosages of 30, 50, and $70 \mathrm{mg} /$ day for 4 weeks, using physician-evaluated ADHD-RS-IV total scores. Secondary study objectives were to assess the efficacy of the three LDX dosages, relative to placebo, using the Conners' Third Edition (Japanese version) Parent Rating Scale (Conners 3), Clinical Global Impression Improvement (CGI-I) scale, Clinical Global Impression Severity (CGI-S) scale, and Parent Global Assessment (PGA) scale; and to evaluate LDX safety according to the incidence of treatment-emergent adverse events (TEAEs).

\section{Study design}

This was a comparative study consisting of four periods: a 1- to 4-week screening period; a 4-week treatment period (double-blind); 
a 1-week placebo period (single-blind); and a 1-week follow-up period, equaling 7-10 weeks in total, although some patients entered a long-term extension study and did not enter the 1-week follow-up period (Fig. 1). LDX at doses of 30, 50, and $70 \mathrm{mg}$ or placebo were administered. To maintain blinding, all investigational products were supplied as white capsules identical in size, weight, and shape. During the 4-week double-blind treatment period, patients started LDX at a dosage of $30 \mathrm{mg}$ once daily. The dosage was uptitrated in increments of $20 \mathrm{mg}$ at weekly intervals until patients reached their assigned fixed dosage. In the 1-week single-blind phase, all patients regardless of initial study medication assignment received placebo once daily in the morning.

LDX efficacy was assessed by means of the ADHD-RS-IV Japanese version, Conners 3, CGI-I, CGI-S, and PGA. LDX safety was evaluated at each study visit by means of a medical examination for TEAEs, bodyweight, blood pressure, ECG, pulse rate, and laboratory test values, and through completion of the Columbia Suicide Severity Rating Scale and Dependence Questionnaires.

\section{Study assessments}

The ADHD RS-IV used was a Japanese language translation composed of 18 items of the DSM-IV-Text Revision (Dupaul et al. 1998; Ichikawa and Tanaka 2008; Ohnishi et al. 2010; Tani et al. 2010). At each study visit, investigators evaluated patients' ADHD symptoms based on a patient's home and classroom behavior by assessing each item in the ADHD RS-IV using a 4-grade scale: "never or rarely" (0 points), "sometimes" (1), "often" (2), or "very often" (3). Behavior (over the past 6 months at visit 1, and from the previous to current visit at all other time points) was assessed by interviewing a parent or guardian of each patient.

The Conners 3 (Japanese version) is a 110-item assessment scale, which parents or guardians used to assess ADHD-related symptoms from visit 2 onward according to a 4-category scale: "Not true at all. It never (or seldom) happened" (0 points); "Just a little true. It happened occasionally" (1); "Pretty much true. It happened often (or quite a bit)" (2); or "Very much true. It happened very often (very frequently)" (3) (Conners 2008, 2011).

At visits 3-7, investigators assessed the improvement from visit 2 in patients' ADHD symptoms according to 7-grade CGI-I and PGA scales: "very much improved", "much improved", "minimally improved", "no change", "minimally worse", "much worse", or "very much worse". The same applied for the CGI-S scale, which has a 7-category scale: "normal, not at all ill", "borderline ill”, "mildly ill", "moderately ill", "markedly ill", "severely ill", or "extremely ill".

All AEs identified from the time of obtaining consent until visit 8 were investigated, and all AEs reported after the initial dose of randomized study drug were considered TEAEs.

An independent Safety Evaluation Committee reviewed information about patients' baseline characteristics, efficacy and safety data, and findings from the Dependence Questionnaires.

\section{Statistical analyses}

SAS $^{\circledR}$ version 9.2 or above (SAS Institute, Inc., Cary, NC) was used for all statistical analyses, and two-sided $p$-values in the SAS default output were used for the Fisher's exact test.

The sample size for the study was determined based on changes from baseline in ADHD-RS-IV total scores in the LDX 30, 50, and $70 \mathrm{mg}$ groups, and in the placebo group, in non-Japanese patients with ADHD who took part in studies in children (Biederman et al. 2007) and adolescents (Findling et al. 2011). Based on results of a Japanese study of atomoxetine (Takahashi et al. 2009), the proportions of patients considered appropriate for enrollment in the current trial were $85 \%$ (age 6-12 years) and 15\% (age 13-17 years). The effect sizes relative to the placebo group were estimated to be $1.15,1.30$, and 1.50 for the LDX $30 \mathrm{mg}, 50 \mathrm{mg}$, and $70 \mathrm{mg}$ groups, respectively, in the overall study population ranging from age 6 to 17 years. The sample size required for a 2 -sample $t$-test was

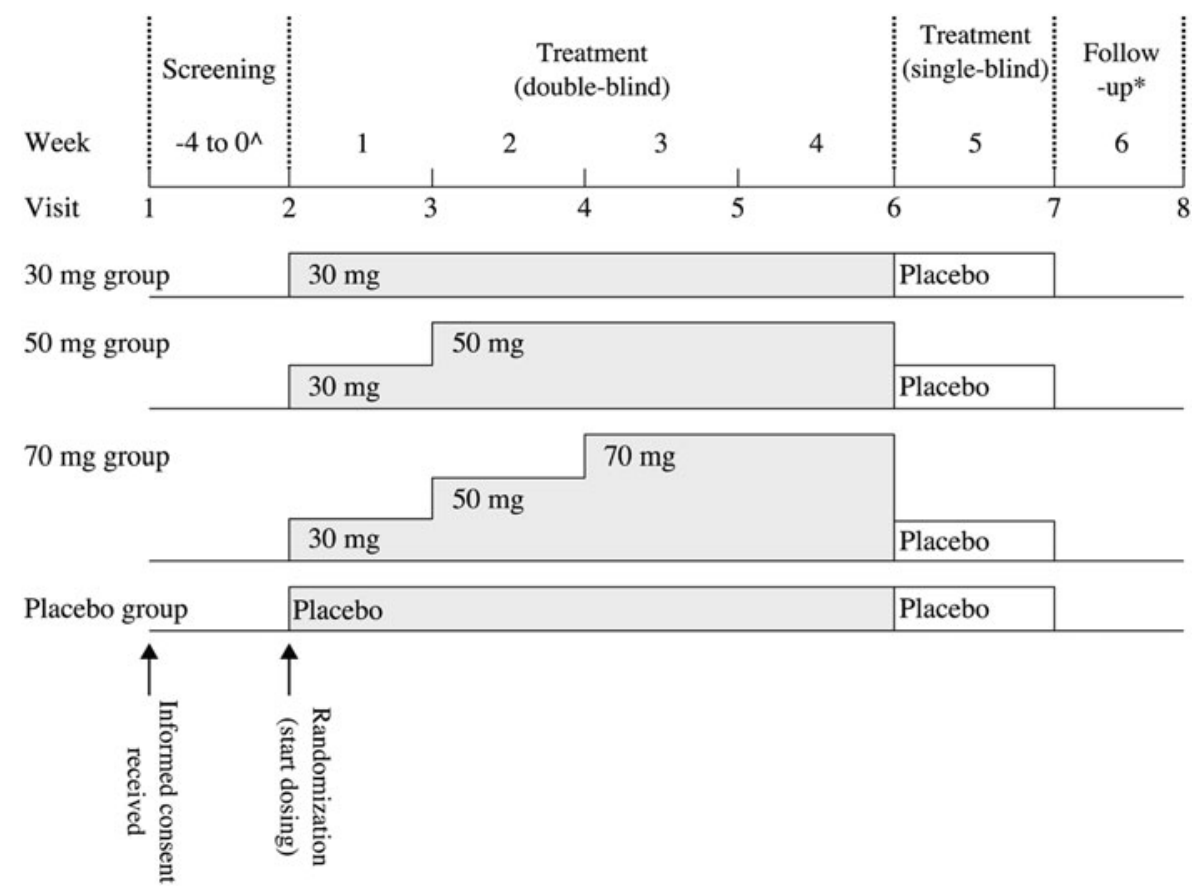

FIG. 1. Study design ^Appropriate only: The duration of the screening period was $1-4$ weeks. * Some patients entered a long-term extension study and did not enter the 1-week follow-up period. 
calculated, considering multiplicity in pairwise comparisons between each LDX dosage group and the placebo group in analysis of the primary endpoint. To adjust for multiplicity arising from pairwise comparisons, the serial gatekeeping method was applied to two families of hypotheses. The first family of hypotheses constituted pairwise comparisons between the LDX $70 \mathrm{mg}$ group and the placebo group, and between the LDX $50 \mathrm{mg}$ group and the placebo group; the second family of hypotheses constituted pairwise comparison between the LDX $30 \mathrm{mg}$ group and the placebo group. Only if both pairwise comparisons in the first family of hypotheses showed significant differences with a two-sided significance level was the pairwise comparison in the second family of hypotheses performed at a two-sided significance level of 0.05. A sample size required to detect superiority for LDX versus placebo with a power of $\geq 90 \%$ (by both tests for the first family of hypotheses), and to detect superiority with a power of $\geq 90 \%$ (by a test for the second family of hypotheses), by controlling the overall significance level below $5 \%$ by serial gatekeeping, was estimated. The required sample size was calculated as 17 patients per group. Assuming that about $10 \%$ of patients would drop out of the primary efficacy analysis, the target sample size was determined as 19 patients per group (i.e., 76 patients overall). The full-analysis (i.e., modified intent-to-treat [mITT]) population, which was the primary population used for efficacy analyses, comprised all randomized patients except: patients with major GCP noncompliance (e.g., informed consent not given, attendance at a center without Institutional Review Board review); patients not receiving the study treatment; or patients with no observations (i.e., the ADHD RS-IV total score, the primary study endpoint, was not evaluated at baseline or from the start of study drug dosing to visit 6).

The per-protocol population included all randomized patients in the m-ITT population who did not meet any of the following conditions: ineligibility (e.g., patients with any protocol viola- tions); treatment violation (e.g., patients with procedural violations associated with concomitant drugs or therapies); noncompliance (e.g., patients whose treatment period compliance was $<80 \%$ or $>120 \%$ ); or inadequate follow-up (e.g., patients withdrawn from the study by visit 6 ).

The primary efficacy endpoint was the change in the ADHD-RSIV total score from baseline (visit 2) to 4 weeks (visit 6). Using all data obtained at visits 3-6, the mixed-effects model repeated measures (MMRM) included dosage group, time point, and the interaction between dosage group and time point as fixed effects, and ADHD RS-IV total score at baseline $(<40, \geq 40)$ and age category (6-9 years, $10-12$ years, and $13-17$ years) as covariates. The MMRM did not assume a specific covariance structure for error variance. If the algorithm did not converge in the above model, heterogeneous autoregression, heterogeneous compound symmetry, compound symmetry, and variance component were selected in this order as the covariance structure of errors for the MMRM. Under the MMRM, adjusted mean differences between dosage groups and 95\% confidence intervals (CIs) and $p$-values were calculated. Using the MMRM, pairwise comparisons were performed between each LDX dosage group and the placebo group regarding the primary endpoint. As described above, to adjust for multiplicity arising from pairwise comparisons, the serial gatekeeping method was applied.

\section{Results}

\section{Study population}

A total of 103 patients were registered to undergo screening, of whom 76 patients entered the double-blind treatment period (Fig. 2). Of these 76 patients, five were withdrawn because of AEs ( $n=2$ : LDX $70 \mathrm{mg}$ group, $n=1$ : LDX $50 \mathrm{mg}$ group), ineligibility ( $n=1$ : LDX $70 \mathrm{mg}$ group), or lack of efficacy $(n=1$ : LDX $30 \mathrm{mg}$

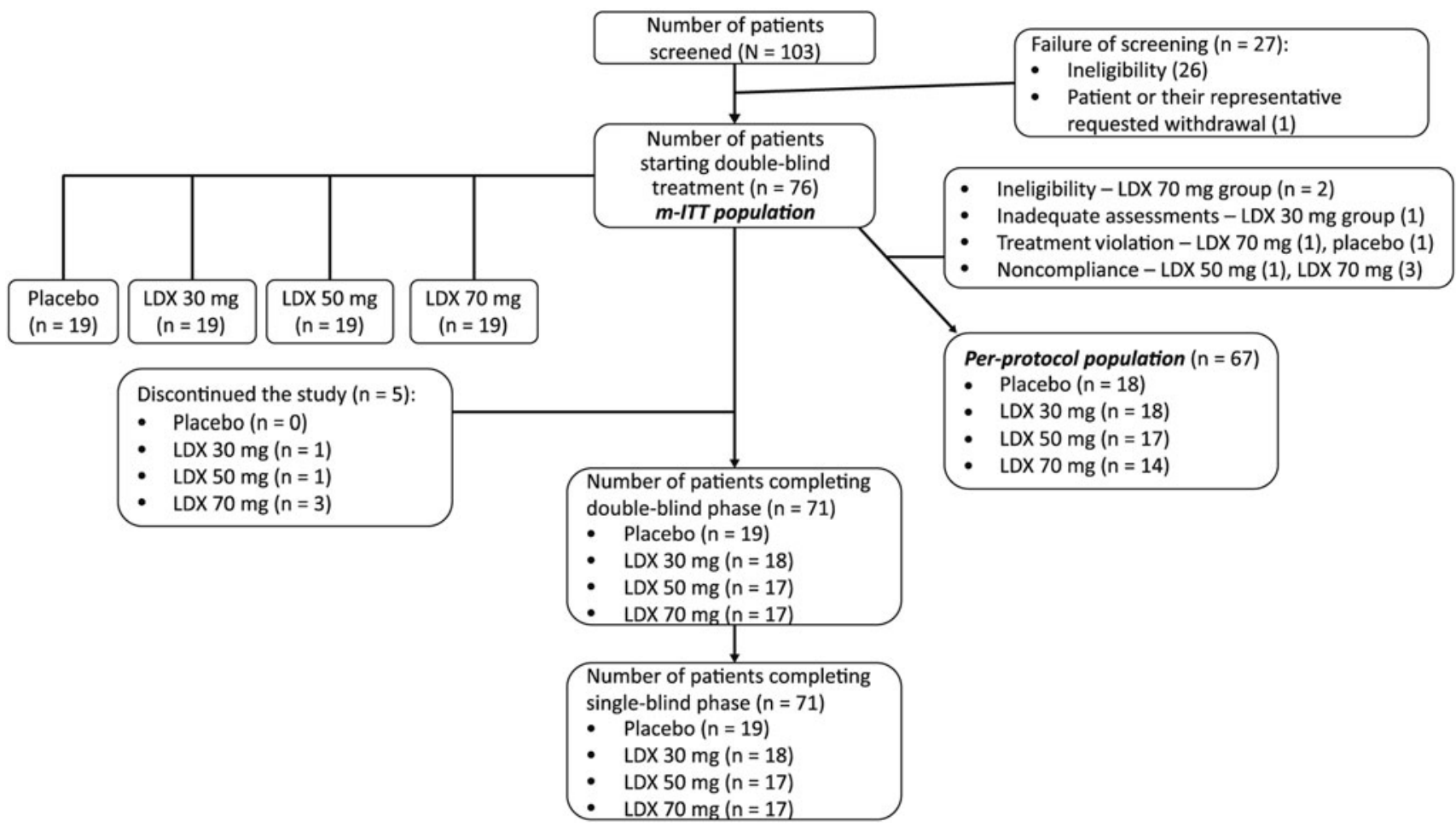

FIG. 2. Patient disposition according to treatment group. LDX, lisdexamfetamine; m-ITT, modified intention-to-treat. 
group). Thus, 71 patients completed the double-blind treatment period, of which all 71 completed the single-blind placebo period.

\section{Patient demographics}

Baseline characteristics of the m-ITT population are shown in Table 1. There were no major differences between treatment groups, except as regards previous medical conditions and ADHD subtype. More placebo recipients than LDX-treated patients had previous medical conditions, and fewer placebo recipients than LDX-treated patients had ADHD of the predominantly inattentive subtype. In all study groups, boys outnumbered girls and consisted $72.2 \%-90.0 \%$ of each group's population. Overall mean age was 9.9-10.1 years, and $60.0 \%-66.7 \%$ of patients had an ADHD-RS-IV total score $<40$ at baseline. The mean baseline ADHD-RS-IV total score was 37.1-38.1. Approximately three-quarters of patients in the LDX $50 \mathrm{mg}$ and placebo groups had combined subtype ADHD, compared with about half of patients in the LDX $30 \mathrm{mg}$ and LDX $70 \mathrm{mg}$ groups. ADHD of the predominantly hyperactive/impulsive subtype was evident in two placebo recipients only.

\section{ADHD-RS-IV scores}

Adjusted mean changes from baseline in the ADHD-RS-IV total score in the m-ITT population are shown in Figure 3. For all three LDX dosages, and at all time points from week 1 to 4 , least-squares mean (LSM) differences for LDX versus placebo in ADHD-RS-IV total score changes from baseline were statistically significant. Differences in changes from baseline to week 4 in the ADHD-RSIV total score between treatment groups and placebo were -13.60 in the LDX $30 \mathrm{mg}$ group ( $p<0.0001$ vs. placebo), -15.32 in the LDX $50 \mathrm{mg}$ group ( $p<0.0001$ vs. placebo), and -13.69 in the LDX $70 \mathrm{mg}$ group $(p<0.0001$ vs. placebo).

\section{Secondary study endpoints}

In the LDX 30, 50, and $70 \mathrm{mg}$ dosage groups, LSM differences (LDX versus placebo) in changes from baseline to week 4 for ADHD-RS-IV inattention subscale scores $(-7.69,-10.66$, and -9.05 , respectively; all $p<0.0001$ vs. placebo) and for hyperac- tivity/impulsivity subscale scores $(-6.25[p=0.0005$ vs. placebo], -5.13 [ $p=0.0046$ vs. placebo], and -5.21 [ $p=0.0038$ vs. placebo], respectively) were statistically significant (Table 2 ).

At weeks 3 and 4, LSM differences (LDX vs. placebo) for changes from baseline in all Conners 3 subscale scores (except the CD subscale score) were statistically significant for all LDX dosages. At week 4, LSM differences for changes from baseline in Conners 3 inattention plus hyperactivity/impulsivity scores were -9.96 for LDX $30 \mathrm{mg}$ ( $p=0.0076$ vs. placebo), -12.41 for LDX $50 \mathrm{mg}$ ( $p=0.0015$ vs. placebo), and -10.04 for LDX $70 \mathrm{mg}$ ( $p=0.0082$ vs. placebo; Table 3$)$.

At all study visits from weeks 1 to 4 (except for the LDX $70 \mathrm{mg}$ group at weeks 1 and 2 and the LDX $50 \mathrm{mg}$ group at week 1), the proportions of patients with improvement on the CGI-I scale (i.e., a score of "much improved" or "very much improved") were significantly higher in LDX groups than in the placebo group. At week $4,61 \%-71 \%$ of patients treated with LDX, compared with $11 \%$ of placebo recipients ( $p \leq 0.0019)$, had CGI-I improvement (Fig. 4).

At all study visits from weeks 1 to 4 , the proportions of patients with improvement on the CGI-S scale (i.e., a score of "normal, not at all ill" or "borderline ill") were greater in LDX groups than in the placebo group. At week 4, 5.6\%-11.8\% of patients treated with LDX, compared with $0.0 \%$ of patients treated with placebo, had CGI-S improvement.

At all study visits from weeks 1 to 4 (except for the LDX $70 \mathrm{mg}$ group at week 1), the proportions of patients with improvement on the PGA scale (i.e., a score of "much improved" or "very much improved") were significantly higher in LDX groups than in the placebo group. At week 4, 56\%-65\% of patients treated with LDX, compared with $16 \%$ of placebo recipients $(p \leq 0.0170)$, had PGA improvement (Fig. 5).

\section{Adverse events}

In the overall safety population $(n=76), 45$ patients treated with LDX experienced a total of 119 TEAEs, comprising 13 patients in the LDX $30 \mathrm{mg}$ group (34 events), 18 patients in the $50 \mathrm{mg}$ group (53 events), and 14 patients in the $70 \mathrm{mg}$ group (32 events). Eight patients in the placebo group $(42.1 \%)$ had 17 TEAEs in total. There

Table 1. Baseline Characteristics of the Modified Intent-to-Treat Population

\begin{tabular}{|c|c|c|c|c|c|}
\hline Characteristic & $\begin{array}{l}\text { Placebo } \\
(\mathrm{n}=19)\end{array}$ & $\begin{array}{l}\text { LDX } 30 m g \\
\quad(\mathrm{n}=19)\end{array}$ & $\begin{array}{l}L D X 50 m g \\
\quad(\mathrm{n}=18)\end{array}$ & $\begin{array}{l}L D X 70 \mathrm{mg} \\
\quad(\mathrm{n}=20)\end{array}$ & $\mathrm{p}$ value \\
\hline Male gender, $n(\%)$ & $16(84.2)$ & $16(84.2)$ & $13(72.2)$ & $18(90.0)$ & $0.5576^{\mathrm{a}}$ \\
\hline Age, years; mean (SD) & $9.9(2.7)$ & $10.1(3.1)$ & $10.0(2.9)$ & $10.1(2.5)$ & $0.9963^{\mathrm{b}}$ \\
\hline Height, cm; mean (SD) & $138.2(15.5)$ & $139.2(17.4)$ & $135.0(13.5)$ & $138.9(13.7)$ & $0.8183^{\mathrm{b}}$ \\
\hline Bodyweight, $\mathrm{kg}$; mean (SD) & $34.9(12.3)$ & $38.5(15.9)$ & $33.1(11.2)$ & $35.8(11.7)$ & $0.6416^{\mathrm{b}}$ \\
\hline Previous medical conditions; $n$ (\% pts) & $8(42.1)$ & $2(10.5)$ & $5(27.8)$ & $3(15.0)$ & $0.1091^{\mathrm{a}}$ \\
\hline Concurrent medical conditions; $n$ (\% pts) & $14(73.7)$ & $14(73.7)$ & $12(66.7)$ & $17(85.0)$ & $0.6216^{\mathrm{a}}$ \\
\hline Previous drug treatment; $n$ (\% pts) & $6(31.6)$ & $7(36.8)$ & $6(33.3)$ & $5(25.0)$ & $0.9024^{\mathrm{a}}$ \\
\hline ADHD subtype; $n$ (\% pts) & & & & & $0.1217^{\mathrm{a}}$ \\
\hline Combined & $14(73.7)$ & $10(52.6)$ & $13(72.2)$ & $11(55.0)$ & \\
\hline Predominantly inattentive & $3(15.8)$ & $9(47.4)$ & $5(27.8)$ & $9(45.0)$ & \\
\hline Predominantly hyperactive/impulsive & $2(10.5)$ & $0(0.0)$ & $0(0.0)$ & $0(0.0)$ & \\
\hline ADHD-RS-IV total score $<40$ at baseline; $n$ ( $\%$ pts) & $12(63.2)$ & $12(63.2)$ & $12(66.7)$ & $12(60.0)$ & $0.9610^{\mathrm{b}}$ \\
\hline ADHD-RS-IV total score $\geq 40$ at baseline; $n$ ( $\%$ pts) & $7(36.8)$ & $7(36.8)$ & $6(33.3)$ & $8(40.0)$ & \\
\hline Baseline ADHD-RS-IV total score; mean (SD) & $37.9(7.4)$ & $38.1(6.7)$ & $37.1(6.9)$ & $37.2(7.8)$ & \\
\hline
\end{tabular}

${ }^{a}$ Fisher's exact test.

${ }^{\mathrm{b}}$ One-way analysis of variance.

ADHD, attention-deficit/hyperactivity disorder; LDX, lisdexamfetamine; m-ITT, modified intent-to-treat; pts, patients; SD, standard deviation. 


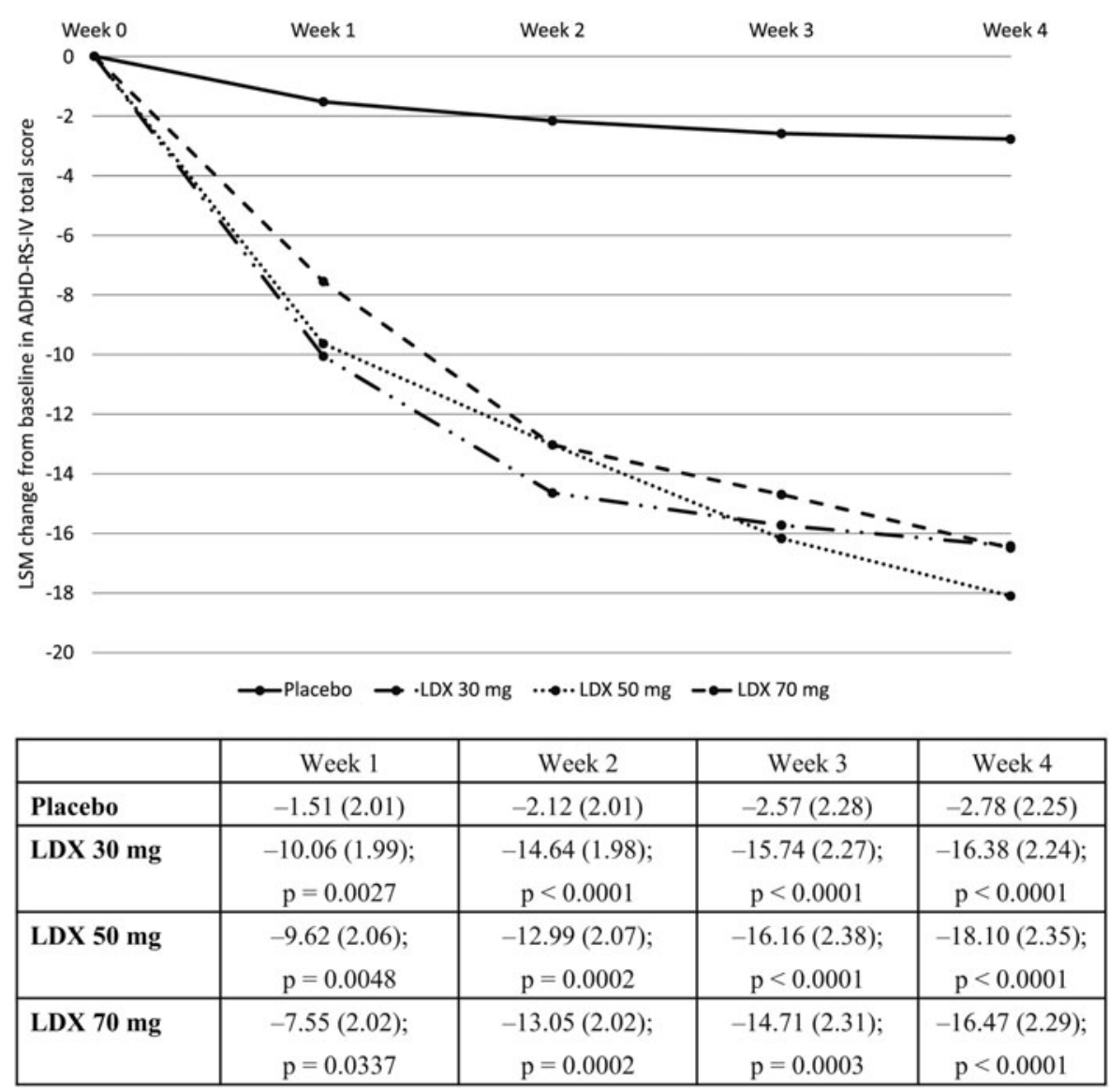

FIG. 3. Time course of change from baseline in ADHD-RS-IV total score: Adjusted mean (standard error). From week 1 onward $p<0.05$ to $p<0.0001$ versus placebo for scores in all LDX groups. LDX, lisdexamfetamine; LSM, least squares mean; MMRM, mixedeffects model repeated measures MMRM analysis. Fixed effect-treatment group, time point; Interaction effect, treatment group and time point; Covariate, baseline ADHD-RS-IV total score ( $<40, \geq 40)$, age (6-9 years, 10-12 years, 13-17 years); Covariance structureunstructured.

were no deaths, serious TEAEs, or severe TEAEs during the study. Four patients experienced five moderate TEAEs: influenza (placebo), decreased appetite (LDX $30 \mathrm{mg}$ ), weight decrease (LDX $30 \mathrm{mg}$ ), hand fracture (LDX $50 \mathrm{mg}$ ), and insomnia (LDX $70 \mathrm{mg}$ ). TEAEs led to treatment discontinuation for one patient in the LDX
$50 \mathrm{mg}$ group (mild insomnia and mild headache), and two patients in the LDX $70 \mathrm{mg}$ group (mild nausea, $n=1$; moderate insomnia, $n=1)$.

TEAEs occurring with an incidence of $\geq 10 \%$ in any study group are listed in Table 4. The most frequent TEAEs were decreased

Table 2. Changes from Baseline to Week 4 in ADHD-RS-IV Subscale Scores (m-ITT Population)

\begin{tabular}{|c|c|c|c|c|c|}
\hline Time point (week) & $\begin{array}{l}\text { Treatment } \\
\text { group }\end{array}$ & $\begin{array}{l}\text { Number } \\
\text { of pts }\end{array}$ & $\begin{array}{c}\text { Change from } \\
\text { baseline; LSM (SE) }\end{array}$ & $\begin{array}{c}\text { Difference of LSM; } \\
\text { LDX vs. placebo }(95 \% \text { CI })\end{array}$ & $\mathrm{p}$ value \\
\hline \multicolumn{6}{|c|}{ Inattention subscale score } \\
\hline \multirow[t]{4}{*}{ Week 4} & Placebo & 19 & $-0.57(1.25)$ & & \\
\hline & LDX $30 \mathrm{mg}$ & 18 & $-8.26(1.23)$ & $-7.69(-11.17$ to -4.22$)$ & $<0.0001$ \\
\hline & LDX $50 \mathrm{mg}$ & 17 & $-11.23(1.28)$ & $-10.66(-14.20$ to -7.12$)$ & $<0.0001$ \\
\hline & LDX $70 \mathrm{mg}$ & 17 & $-9.62(1.27)$ & $-9.05(-12.61$ to -5.48$)$ & $<0.0001$ \\
\hline \multicolumn{6}{|c|}{ Hyperactivity/impulsivity subscale score } \\
\hline \multirow[t]{4}{*}{ Week 4} & Placebo & 19 & $-1.89(1.21)$ & & \\
\hline & LDX $30 \mathrm{mg}$ & 18 & $-8.14(1.21)$ & $-6.25(-9.64$ to -2.86$)$ & 0.0005 \\
\hline & LDX $50 \mathrm{mg}$ & 17 & $-7.02(1.27)$ & $-5.13(-8.62$ to -1.65$)$ & 0.0046 \\
\hline & LDX $70 \mathrm{mg}$ & 17 & $-7.10(1.26)$ & $-5.21(-8.68$ to -1.74$)$ & 0.0038 \\
\hline
\end{tabular}

MMRM analysis: Fixed effect—-treatment group, time point; Interaction effect—treatment group and time point; Covariate—each baseline ADHD-RSIV subscale score, age (6-9 years, 10-12 years, 13-17 years); Covariance structure-unstructured.

ADHD, attention-deficit/hyperactivity disorder; CI, confidence interval; LDX, lisdexamfetamine; LSM, least-squares mean; m-ITT, modified intent-totreat; MMRM, mixed-effects model repeated measures; pts, patients; SE, standard error. 
Table 3. Changes from Baseline to Week 4 in Conners 3 Inattention Plus Hyperactivity/Impulsivity Subscale Scores (M-ITT Population)

\begin{tabular}{|c|c|c|c|c|c|}
\hline Time point (week) & Treatment group & $\begin{array}{l}\text { Number } \\
\text { of pts }\end{array}$ & $\begin{array}{c}\text { Change from } \\
\text { baseline; LSM (SE) }\end{array}$ & $\begin{array}{c}\text { Difference of LSM; } \\
\text { LDX vs. placebo }(95 \% \text { CI) }\end{array}$ & $\mathrm{p}$ value \\
\hline \multirow[t]{4}{*}{ Week 4} & Placebo & 19 & $-2.33(2.63)$ & & \\
\hline & LDX $30 \mathrm{mg}$ & 18 & $-12.29(2.59)$ & $-9.96(-17.18$ to -2.74$)$ & 0.0076 \\
\hline & LDX $50 \mathrm{mg}$ & 17 & $-14.74(2.71)$ & $-12.41(-19.87$ to -4.96$)$ & 0.0015 \\
\hline & LDX $70 \mathrm{mg}$ & 17 & $-12.36(2.66)$ & $-10.04(-17.39$ to -2.68$)$ & 0.0082 \\
\hline
\end{tabular}

MMRM analysis: Fixed effect-treatment group, time point; Interaction effect-treatment group and time point; Covariate-baseline Conners 3 inattention plus hyperactivity/impulsivity subscale score, age (6-9 years, 10-12 years, 13-17 years); Covariance structure-unstructured.

CI, confidence interval; LDX, lisdexamfetamine; LSM, least-squares mean; m-ITT, modified intent-to-treat; MMRM, mixed-effects model repeated measures; pts, patients; SE, standard error.

appetite $(47.4 \%-77.8 \%$ of LDX-treated patients vs. $0.0 \%$ of placebo recipients), headache $(5.0 \%-38.9 \%$ vs. $0.0 \%)$, initial insomnia $(10.5 \%-27.8 \%$ vs. $0.0 \%)$, and nasopharyngitis $(5.0 \%-22.2 \%$ vs. $21.1 \%$ ). No cases of nasopharyngitis in LDX-treated patients were considered as treatment related. Most TEAEs occurred within 1 week of starting LDX treatment: that is, $10 / 19$ patients $(52.6 \%)$ in the $30 \mathrm{mg}$ group, $16 / 18$ patients $(88.9 \%)$ in the $50 \mathrm{mg}$ group, and $9 / 20$ patients $(45.0 \%)$ in the $70 \mathrm{mg}$ group. No new AEs were noted $>4$ weeks after starting treatment.

No clinically significant changes in laboratory parameters were observed during the study. No major changes were noted in systolic blood pressure before and after LDX administration in all dosage groups. From baseline to final study evaluation, mean diastolic blood pressure decreased by $2.54 \mathrm{~mm} \mathrm{Hg}$ in the placebo group, and increased by $2.51 \mathrm{~mm} \mathrm{Hg}$ in the LDX $30 \mathrm{mg}$ group, $7.76 \mathrm{~mm} \mathrm{Hg}$ in the LDX $50 \mathrm{mg}$ group, and $2.65 \mathrm{~mm} \mathrm{Hg}$ in the LDX $70 \mathrm{mg}$ group. Corresponding changes in pulse rate were -3.77 beats $/ \mathrm{min},+7.49$ beats/min, +11.56 beats $/ \mathrm{min}$, and +7.45 beats $/ \mathrm{min}$. No blood pressure or pulse rate changes were deemed to be TEAEs.

Mean bodyweight increased by $0.5 \mathrm{~kg}$ in the placebo group from baseline to final evaluation, whereas decreases were noted in LDX- treated patients: $-1.39 \mathrm{~kg}$ in the $30 \mathrm{mg}$ group, $-1.23 \mathrm{~kg}$ in the $50 \mathrm{mg}$ group, and $-1.68 \mathrm{~kg}$ in the $70 \mathrm{mg}$ group. Four bodyweight changes (weight decrease) were classified as TEAEs and occurred in one patient in the $30 \mathrm{mg}$ group (moderate), two patients in the $50 \mathrm{mg}$ group (mild), and one patient in the $70 \mathrm{mg}$ group (mild).

One patient in the LDX $50 \mathrm{mg}$ group and one patient in the $70 \mathrm{mg}$ group had a QTcF interval $>430$ mseconds at any time point. No patient had a QTcF interval $>500$ mseconds. No ECG abnormalities were regarded as TEAEs.

The independent Safety Evaluation Committee, after reviewing information about baseline characteristics, efficacy and safety data, and findings from Dependence Questionnaires, concluded that there were no cases of suspected drug dependence.

\section{Discussion}

A feature of this multicenter, randomized, double-blind, fixeddose study of LDX in Japanese pediatric patients with ADHD was that the primary study endpoint, the change in the ADHD-RS-IV total score from baseline to week 4, was statistically significantly greater $(p<0.0001)$ in all three LDX dosage groups relative to

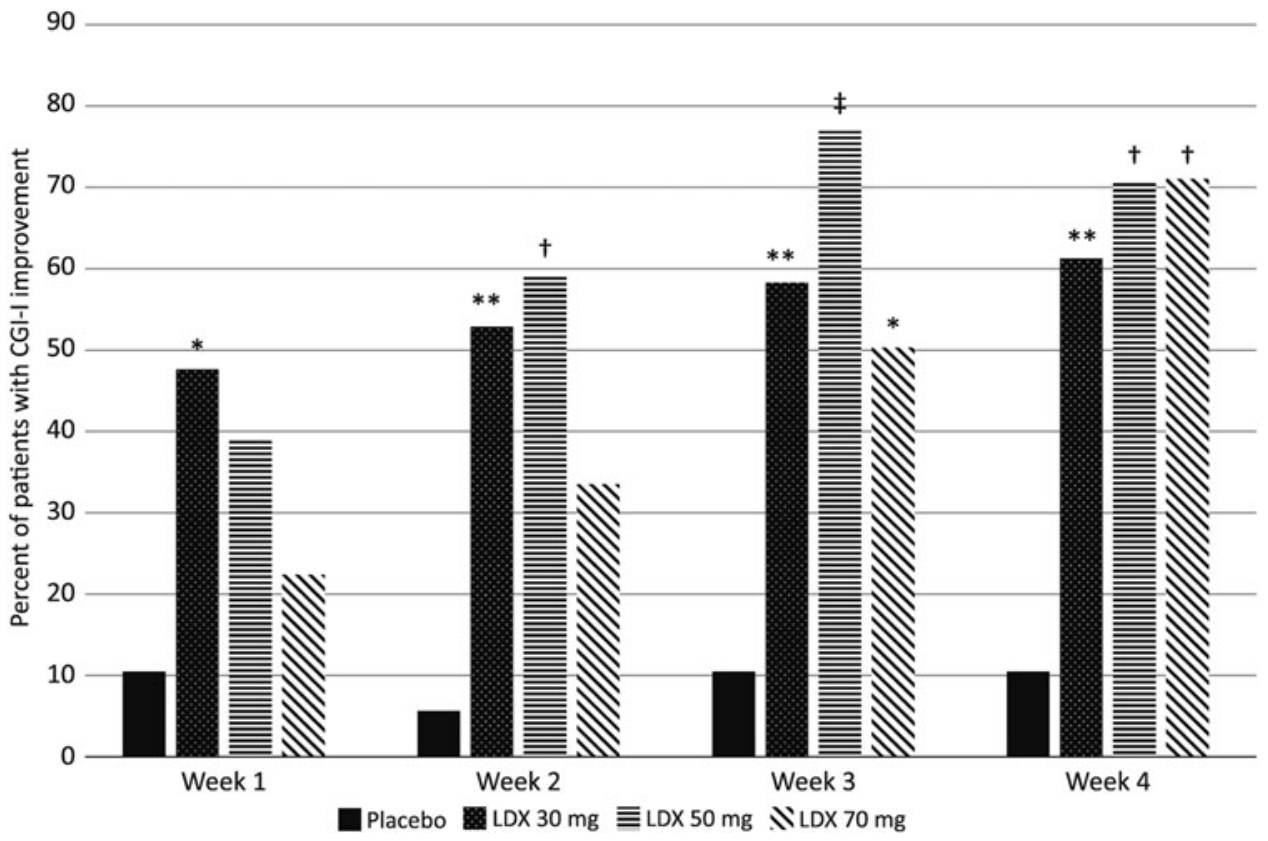

FIG. 4. Proportion of patients with improvement on the GCI-I scale. Statistical significance versus placebo using Fisher's exact test: ${ }^{*} p<0.05 ; * * p<0.01 ;{ }^{\dagger} p<0.001 ;{ }^{\star} p \leq 0.0001$. GCI-I, clinical global impression-improvement; LDX, lisdexamfetamine. 


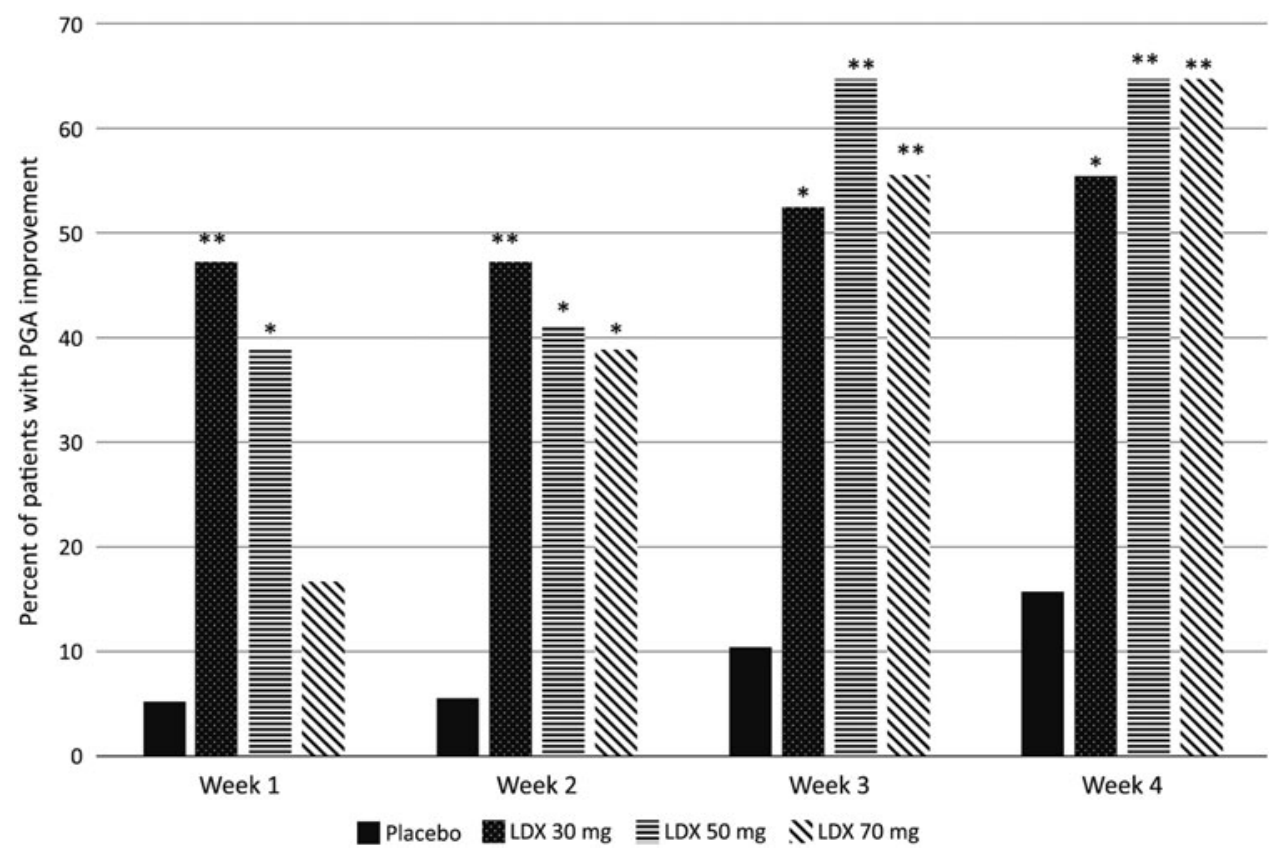

FIG. 5. Proportion of patients with improvement on the PGA scale. Statistical significance versus placebo using Fisher's exact test: $* p<0.05 ; * *<<0.01$. LDX, lisdexamfetamine; PGA, parent global assessment.

placebo. At all assessment points during the trial, improvements (decreases) in the ADHD-RS-IV total score, inattention subscale score, and hyperactivity/impulsivity subscale score were statistically significantly greater in all three LDX groups than in the placebo group, except for the change in the hyperactivity/impulsivity subscale score at week 1 in the LDX $70 \mathrm{mg}$ group $(p=0.1099)$.

Overall, LDX was generally well tolerated, and the AE profile was similar to that previously associated with stimulant therapy in non-Japanese children and adolescents with ADHD (Clavenna and Bonati 2017; Groenman et al. 2017). The most frequent TEAEs were decreased appetite $(47.4 \%-77.8 \%$ of LDX recipients), headache $(5.0 \%-38.9 \%)$, and initial insomnia $(10.5 \%-27.8 \%)$. There were no deaths or serious or severe TEAEs during the trial, and most TEAEs were mild. Four patients had a total of five moderate
TEAEs which, in LDX-treated patients, comprised decreased appetite, weight decrease, hand fracture, and insomnia. No blood pressure or pulse rate changes were considered TEAEs, only four patients had bodyweight changes (weight decrease) considered TEAEs, and no patient had a QTcF interval >500 mseconds.

Efficacy data from our trial compare favorably with, and are at least similar to, data from large European and U.S. studies of LDX in children and adolescents with ADHD (Biederman et al. 2007; Wigal et al. 2009, 2010; Findling et al. 2011; Dittmann et al. 2013, 2014; Stuhec et al. 2015; Coghill et al. 2017; Newcorn et al. 2017).

In Europe, for example, a direct head-to-head study in 267 children and adolescents with ADHD and previous inadequate response to methylphenidate showed that LDX produced a significantly faster clinical response than atomoxetine and was associated

Table 4. Treatment-Emergent Adverse Events Occurring with an Incidence of $\geq 10 \%$

\begin{tabular}{|c|c|c|c|c|}
\hline Preferred term & $\begin{array}{c}\text { Placebo } \\
(\mathrm{n}=19)\end{array}$ & $\begin{array}{c}L D X 30 m g \\
\quad(\mathrm{n}=19)\end{array}$ & $\begin{array}{l}L D X 50 m g \\
\quad(\mathrm{n}=18)\end{array}$ & $\begin{array}{l}L D X 70 m g \\
\quad(\mathrm{n}=20)\end{array}$ \\
\hline Patients with any TEAEs & $8(42.1)$ & $13(68.4)$ & $18(100.0)$ & $14(70.0)$ \\
\hline Nasopharyngitis & $4(21.1)$ & $2(10.5)$ & $4(22.2)$ & $1(5.0)$ \\
\hline Decreased appetite & $0(0.0)$ & $9(47.4)$ & $14(77.8)$ & $11(55.0)$ \\
\hline Initial insomnia & $0(0.0)$ & $2(10.5)$ & $5(27.8)$ & $5(25.0)$ \\
\hline Insomnia & $0(0.0)$ & $0(0.0)$ & $3(16.7)$ & $1(5.0)$ \\
\hline Headache & $0(0.0)$ & $2(10.5)$ & $7(38.9)$ & $1(5.0)$ \\
\hline Excessive eye blinking & $0(0.0)$ & $0(0.0)$ & $2(11.1)$ & $0(0.0)$ \\
\hline Abdominal pain & $1(5.3)$ & $1(5.3)$ & $1(5.6)$ & $2(10.0)$ \\
\hline Nausea & $0(0.0)$ & $0(0.0)$ & $1(5.6)$ & $2(10.0)$ \\
\hline Stomatitis & $1(5.3)$ & $2(10.5)$ & $1(5.6)$ & $0(0.0)$ \\
\hline Constipation & $0(0.0)$ & $2(10.5)$ & $0(0.0)$ & $0(0.0)$ \\
\hline Feeling abnormal & $0(0.0)$ & $0(0.0)$ & $2(11.1)$ & $0(0.0)$ \\
\hline Weight decrease & $0(0.0)$ & $1(5.3)$ & $2(11.1)$ & $1(5.0)$ \\
\hline
\end{tabular}

Data shown are number $(\%)$ of patients from the safety population with TEAEs.

TEAEs, treatment-emergent adverse events. 
with significantly greater treatment response rates than atomoxetine across all response criteria assessed (Dittmann et al. 2013). A large meta-analysis of 28 studies involving children and adolescents with ADHD demonstrated that, relative to placebo, the comparative efficacy of LDX in reducing ADHD symptoms (standardized mean difference -1.28$)$ was greater than that for bupropion $(-0.32)$, atomoxetine (-0.68), and methylphenidate $(-0.75)$ (Stuhec et al. 2015). More recently, a 2-year analysis of LDX safety and efficacy in children and adolescents with ADHD revealed that LDX was generally well tolerated; the most frequent TEAEs tended to occur early during treatment, then decline in incidence, and no new safety signals emerged (Coghill et al. 2017). Regarding efficacy, LDX produced a consistent and sustained improvement in ADHD symptoms over the 2-year observation period, at which time $77.3 \%$ of study participants had a treatment response (CGI-I score $\leq 2$, and $\geq 50 \%$ decrease from baseline in the ADHD-RS-IV total score).

Studies conducted in the United States also demonstrated favorable results with LDX. For example, a phase III trial in 290 children (6-12 years of age) with ADHD revealed that LDX 30$70 \mathrm{mg} /$ day versus placebo for 4 weeks significantly $(p<0.001)$ improved ADHD-RS-IV and Conners' Parent Rating Scale scores; LDX efficacy was evident by week 1 of treatment and was maintained throughout the day (i.e., up until about $6 \mathrm{pm}$ in the evening) (Biederman et al. 2007). In 314 adolescents (13-17 years of age) with ADHD, LDX $30-70 \mathrm{mg} /$ day for 4 weeks produced significantly (all $p \leq 0.0056$ ) greater LSM changes from baseline in ADHD-RS-IV total scores than placebo $(30 \mathrm{mg},-18.3 ; 50 \mathrm{mg}$, -21.1; $70 \mathrm{mg},-20.7$; placebo, -12.8); CGI-I improvement was evident in $69.1 \%$ of LDX-treated patients compared with $39.5 \%$ of placebo recipients $(p<0.0001)$ (Findling et al. 2011). In addition, two randomized, double-blind, placebo-controlled, head-to-head trials also directly compared the efficacy of LDX with OROS methylphenidate in adolescents 13-17 years of age with ADHD (Newcorn et al. 2017). In a flexible-dose study of LDX 30$70 \mathrm{mg} /$ day versus OROS methylphenidate $18-72 \mathrm{mg} /$ day, no significant difference in effect size was evident between the two stimulants, whereas in a forced-dose study of LDX $70 \mathrm{mg} /$ day versus OROS methylphenidate $72 \mathrm{mg} /$ day, the effect size significantly favored LDX ( $p=0.0013)$.

Furthermore, in the United States, Wigal and colleagues conducted a laboratory school study of LDX $30-70 \mathrm{mg}$ /day for 4 weeks in 117 children (6-12 years of age) with ADHD (Wigal et al. 2009, 2010). LDX showed significantly greater efficacy than placebo ( $p<0.005$ ), with medium to large effect sizes throughout the day, from $\sim 1.5$ to 13.0 hours postdose, on the Swanson, Kotkin, Agler, M-Flynn, and Pelham (SKAMP) deportment and attention subscales, and on the Permanent Product Measure of Performance scale. In a similar laboratory school study, an extended-release formulation of methylphenidate demonstrated a significant effect versus placebo on SKAMP-combined score from 0.75 to 12.0 hours postdose (Wigal et al. 2013).

Findings of the independent Safety Evaluation Committee in the current trial suggest a low risk of substance abuse with LDX. Moreover, a large analysis of nonmedical use of prescription ADHD stimulants, based on the U.S. National Addictions Vigilance Intervention and Prevention Program, revealed that nonmedical use during the previous 30 days was significantly lower for prescription stimulants $(1.3 \%)$ than for opioids $(19.8 \%)$ and sedatives (10.6\%). Among stimulants, rates of nonmedical use per prescription were considerably lower for LDX and OROS methylphenidate (0.9-1.3) than for mixed amphetamine salts (3.2-4.9) and immediate-release methylphenidate (4.7) (Cassidy et al. 2015).

\section{Limitations}

Study limitations include the relatively small scale and short duration of the trial, and the fact that no LDX-treated patients had the predominantly hyperactive/impulsive subtype of ADHD. On the other hand, the study was controlled, well designed, and had statistical rigor. Its limitations create some interesting possibilities for specific future research in Japanese children and adolescents with ADHD. Notably, research into LDX duration of effect and LDX clinical activity after school hours might be pertinent, as might longer-term evaluation of safety topics, such as the potential for substance abuse, growth retardation in children, and the cardiovascular safety of stimulants (Hennissen et al. 2017). Studies such as the ADDUCE trial, a 2-year pharmacovigilance investigation at 27 European centers (Inglis et al. 2016), may soon provide valuable information about stimulant safety in non-Japanese individuals. Other factors of potential major relevance to Japanese pediatric patients with ADHD, which may warrant research are: the extent of misuse of stimulant ADHD medications; direct comparative studies of LDX with OROS methylphenidate (particularly regarding duration of effect), atomoxetine, and extended-release guanfacine; and investigation of a possible future clinical role for LDX plus nonstimulant combination therapy in some ADHD patients. Meanwhile, our study provides important data about a novel pharmacotherapeutic option for ADHD, namely LDX, in Japanese pediatric patients. Such data may ultimately facilitate improved management of the disorder in Japanese children and adolescents.

\section{Conclusions}

In summary, the superiority of 4 weeks' administration of LDX at dosages of 30,50 , and $70 \mathrm{mg} /$ day versus placebo was demonstrated in Japanese children and adolescents (6-17 years of age) with ADHD. In line with data from large-scale clinical studies of LDX in ADHD patients from other geographical regions, the current trial identified no major safety or tolerability concerns regarding LDX use in Japanese children and adolescents with ADHD.

\section{Clinical Significance}

The prevalence of ADHD in Japan is high, estimated at 7.7\% in children 4-12 years of age living in the greater Tokyo area. However, only three medicines are approved in Japan for treatment of ADHD: OROS methylphenidate, atomoxetine, and extendedrelease guanfacine. More options are needed to facilitate improved management of the disorder. The reported prolonged clinical effect of LDX of up to 13 hours makes it an interesting option. The major clinical significance of the current multicenter, randomized, double-blind trial is that it confirms, as in European and U.S. pediatric patients with ADHD that LDX is significantly effective and generally well tolerated in Japanese children and adolescents with ADHD. These results signify that LDX may be a viable therapeutic choice to facilitate enhanced ADHD management among Japanese children and adolescents.

\section{Acknowledgments}

The authors wish to thank the patients and investigators who took part in the study.

The authors thank Content Ed Net for writing and editorial assistance in the preparation of this article, with funding from Shionogi \& Co. Ltd, Osaka, Japan, and Shire Development LLC, Lexington, Massachusetts, USA. 


\section{Disclaimer}

This information or content and conclusion are those of authors.

\section{Data Sharing}

Researchers can request access to detailed information about Shionogi's clinical trials, including trial protocols and individual patient data, on the portal site https://clinicalstudydatarequest.com. Sharable information includes data about Shionogi's clinical trials conducted in patients in Japan. The information will become sharable after the medicinal products for which the trials are performed have been approved in Japan. Note that all documents will be provided in Japanese language as they have been prepared in Japanese.

\section{Disclosures}

H.I. received research and funding support from Shionogi \& Co. Ltd. and Shire Development LLC during conduct of the study; receives or has received lecture, article, and other fees from AbbVie GK, Eli Lilly Japan K.K., Hisamitsu Pharmaceutical Co. Inc., Janssen Pharmaceutical K.K., Meiji Seika Pharma Co. Ltd., Otsuka Pharmaceutical Co. Ltd., Shionogi \& Co. Ltd., Shire Development LLC, and Taisho Pharmaceutical Co. Ltd. outside the submitted work.

T.M. received research and funding support from Shionogi \& Co. Ltd. and Shire Development LLC during conduct of the study; receives or has received lecture, consulting, and other fees from Astellas Pharma Inc., Eli Lilly Japan K.K., Otsuka Pharmaceutical Co. Ltd., Shionogi \& Co. Ltd. and Shire Development LLC outside the submitted work.

Y.Y. received research and funding support from Shionogi \& Co. Ltd. and Shire Development LLC during conduct of the study; receives or has received lecture, editorial supervising, and other fees from Eli Lilly Japan K.K., Janssen Pharmaceutical K.K., Otsuka Pharmaceutical Co. Ltd., Shionogi \& Co. Ltd., Shire Development LLC, and Taisho Pharmaceutical Co. Ltd. outside the submitted work

M.F. and A.F. are employees of Shionogi \& Co. Ltd, and own Shionogi shares.

K.S. received research and funding support from Shionogi \& Co. Ltd. and Shire Development LLC during conduct of the study; receives or has received lecture, editorial supervising and other fees from Eli Lilly Japan K.K., Hisamitsu Pharmaceutical Co. Inc., Janssen Pharmaceutical K.K., Meiji Seika Pharma Co. Ltd., Mitsubishi Tanabe Pharma Corporation, Otsuka Pharmaceutical Co. Ltd., Shionogi \& Co. Ltd., Shire Development LLC, Shire Japan K.K., Sumitomo Dainippon Pharma Co. Ltd., Taisho Pharmaceutical Co. Ltd., and Yoshitomiyakuhin Corporation outside the submitted work.

\section{References}

Adler LA, Alperin S, Leon T, Faraone SV: Pharmacokinetic and pharmacodynamic properties of lisdexamfetamine in adults with attention-deficit/hyperactivity disorder. J Child Adolesc Psychopharmacol 27:196-199, 2017.

American Psychiatric Association: Diagnostic and Statistical Manual of Mental Disorders, 5th ed. Arlington, VA: American Psychiatric Publishing; 2013.

Biederman J, Krishnan S, Zhang Y, McGough JJ, Findling RL: Efficacy and tolerability of lisdexamfetamine dimesylate (NRP-104) in children with attention-deficit/hyperactivity disorder: A phase III, multicenter, randomized, double-blind, forced-dose, parallelgroup study. Clin Ther 29;450-463, 2007.

Cassidy TA, McNaughton EC, Varughese S, Russo L, Zulueta M, Butler SF: Nonmedical use of prescription ADHD stimulant medications among adults in a substance abuse treatment population: Early findings from the NAVIPRPO surveillance system. J Atten Disord 19:275-283, 2015.

Catala-Lopez F, Hutton B, Nunez-Beltran A, Page MJ, Ridao M, Macías Saint-Gerons D, Catalá MA, Tabarés-Seisdedos R, Moher D: The pharmacological and non-pharmacological treatment of attention deficit hyperactivity disorder in children and adolescents: A systematic review with network meta-analyses of randomised trials. PLoS One 12:e0180355, 2017.

Clavenna A, Bonati M: Pediatric pharmacoepidemiology - safety and effectiveness of medicines for ADHD. Expert Opin Drug Saf 16; 1335-1345, 2017.

Coghill DR, Banaschewski T, Nagy P, Otero IH, Soutullo C, Yan B, Caballero B, Zuddas A: Long-term safety and efficacy of lisdexamfetamine dimesylate in children and adolescents with ADHD: A phase IV, 2-year, open-label study in Europe. CNS Drugs 31:625638, 2017.

Coghill DR, Caballero B, Sorooshian S, Civil R: A systematic review of the safety of lisdexamfetamine dimesylate. CNS Drugs 28:497511, 2014.

Conners KC (translated by Tanaka Y, Sakamoto R): Conners 3 Japanese version manual. Tokyo, Japan, KanekoShobo, 2011 [Japanese].

Conners KC: Conners 3. Toronto, ON, Multi-Health Systems, Inc., 2008.

Cortese S, Adamo N, Mohr-Jensen C, Hayes AJ, Bhatti S, Carucci S, Del Giovane C, Atkinson LZ, Banaschewski T, Simonoff E, Zuddas A, Barbui C, Purgato M, Steinhausen HC, Shokraneh F, Xia J, Cipriani A, Coghill D; European ADHD Guidelines Group (EAGG): Comparative efficacy and tolerability of pharmacological interventions for attention-deficit/hyperactivity disorder in children, adolescents and adults: Protocol for a systematic review and network meta-analysis. BMJ Open 7:e013967, 2017.

Dittmann RW, Cardo E, Nagy P, Anderson CS, Adeyi B, Caballero B, Hodgkins P, Civil R, Coghill DR: Treatment response and remission in a double-blind, randomized, head-to-head study of lisdexamfetamine dimesylate and atomoxetine in children and adolescents with attentiondeficit hyperactivity disorder. CNS Drugs 28:1059-1069, 2014.

Dittmann RW, Cardo E, Nagy P, Anderson CS, Bloomfield R, Caballero B, Higgins N, Hodgkins P, Lyne A, Civil R, Coghill D: Efficacy and safety of lisdexamfetamine dimesylate and atomoxetine in the treatment of attention-deficit/hyperactivity disorder: A head-to-head, randomized, double-blind, phase IIIb study. CNS Drugs 27:1081-1092, 2013.

Dolder PC, Strajhar P, Vizeli P, Hammann F, Odermatt A, Liechti ME: Pharmacokinetics and pharmacodynamics of lisdexamfetamine compared with d-amphetamine in healthy subjects. Front Pharmacol 8:617, 2017.

Dupaul GJ, Power TJ, Anastopoulos, AD, Reid R: ADHD Rating Scale-IV: Checklists, norms, and clinical interpretation. New York, NY, Guildford Press, 1998.

Findling RL, Childress AC, Cutler AJ, Gasior M, Hamdani M, FerreiraCornwell MC, Squires L: Efficacy and safety of lisdexamfetamine dimesylate in adolescents with attention-deficit/hyperactivity disorder. J Am Acad Child Adolesc Psychiatry 50;395-405, 2011.

Groenman AP, Schweren LJ, Dietrich A, Hoekstra PJ: An update on the safety of psychostimulants for the treatment of attention-deficit/ hyperactivity disorder. Expert Opin Drug Saf 16;455-464, 2017.

Heal DJ, Buckley NW, Gosden J, Slater N, France CP, Hackett D: A preclinical evaluation of the discriminative and reinforcing 
properties of lisdexamfetamine in comparison to D-amfetamine, methylphenidate and modafinil. Neuropharmacology 73:348-358, 2013.

Hennissen L, Bakker MJ, Banaschewski T, Carucci S, Coghill D, Danckaerts M, Dittmann RW, Hollis C, Kovshoff H, McCarthy S, Nagy P, Sonuga-Barke E, Wong IC, Zuddas A, Rosenthal E, Buitelaar JK; ADDUCE consortium: Cardiovascular effects of stimulant and non-stimulant medication for children and adolescents with ADHD: A systematic review and meta-analysis of trials of methylphenidate, amphetamines and atomoxetine. CNS Drugs 31;199215, 2017.

Hutson PH, Pennick M, Secker R: Preclinical pharmacokinetics, pharmacology and toxicology of lisdexamfetamine: A novel damphetamine pro-drug. Neuropharmacology 87;41-50, 2014.

Ichikawa H, Tanaka Y: ADHD Rating Scale-IV: Checklists, norms, and clinical interpretation. Tokyo, Japan, AkashiShoten, 2008 [Japanese].

Inglis SK, Carucci S, Garas P, Häge A, Banaschewski T, Buitelaar JK, Dittmann RW, Falissard B, Hollis C, Kovshoff H, Liddle E, McCarthy S, Nagy P, Neubert A, Rosenthal E, Sonuga-Barke E, Wong I, Zuddas A, Coghill DC; ADDUCE Consortium: Prospective observational study protocol to investigate long-term adverse effects of methylphenidate in children and adolescents with ADHD: The Attention Deficit Hyperactivity Disorder Drugs Use Chronic Effects (ADDUCE) study. BMJ Open 6;e010433, 2016.

Jasinski DR, Krishnan S: Abuse liability and safety of oral lisdexamfetamine dimesylate in individuals with a history of stimulant abuse. J Psychopharmacol 23:419-427, 2009.

Newcorn JH, Nagy P, Childress AC, Frick G, Yan B, Pliszka S: Randomized, double-blind, placebo-controlled acute comparator trials of lisdexamfetamine and extended-release methylphenidate in adolescents with attention-deficit/hyperactivity disorder. CNS Drugs 31:999-1014, 2017.

Ohnishi M, Okada R, Tani I, Nakajima S, Tsujii M: Japanese version of school form of the ADHD-RS: An evaluation of its reliability and validity. Res Dev Disabil 31;1305-1312, 2010.

Ruiz-Goikoetxea M, Cortese S, Aznarez-Sanado M, Magallón S, Alvarez Zallo N, Luis EO, de Castro-Manglano P, Soutullo C, Arrondo G: Risk of unintentional injuries in children and adolescents with ADHD and the impact of ADHD medications: A systematic review and meta-analysis. Neurosci Biobehav Rev 84;6371, 2017.

Special Needs Education Division, the Elementary and Secondary Education Bureau, Japanese Ministry of Education, Culture, Sports, Science, and Technology 2012. Results of research about children/students with probable developmental disabilities who need special educational support in the regular class: December 5, 2012. Available at: www.mext.go.jp/a_menu/shotou/tokubetu/material/ 1328729.htm; (Report) www.mext.go.jp/a_menu/shotou/tokubetu/ material/_icsFiles/afieldfile/2012/12/10/1328729_01.pdf [Accessed 31 May 2018].

Steer C, Froelich J, Soutullo CA, Johnson M, Shaw M: Lisdexamfetamine dimesylate: A new therapeutic option for attentiondeficit hyperactivity disorder. CNS Drugs 26;691-705, 2012.
Stuhec M, Munda B, Svab V, Locatelli I: Comparative efficacy and acceptability of atomoxetine, lisdexamfetamine, bupropion and methylphenidate in treatment of attention deficit hyperactivity disorder in children and adolescents: A meta-analysis with focus on bupropion. J Affect Disord 178;149-159, 2015.

Sulzer D, Sonders MS, Poulsen NW, Galli A: Mechanisms of neurotransmitter release by amphetamines: A review. Prog Neurobiol 75;406-433, 2005.

Takahashi M, Takita Y, Yamazaki K, Hayashi T, Ichikawa H, Kambayashi Y, Koeda T, Oki J, Saito K, Takeshita K, Allen AJ: A randomized, double-blind, placebo-controlled study of atomoxetine in Japanese children and adolescents with attention-deficit/ hyperactivity disorder. J Child Adolesc Psychopharmacol 19:341350, 2009.

Tandon M, Pergjika A: Attention deficit hyperactivity disorder in preschool-age children. Child Adolesc Psychiatr Clin N Am 26; 523-538, 2017.

Tani I, Okada R, Ohnishi M, Nakajima S, Tsujii M: Japanese version of home form of the ADHD-RS: An evaluation of its reliability and validity. Res Dev Disabil 31;1426-1433, 2010.

Taylor E: Attention deficit hyperactivity disorder: Overdiagnosed or diagnoses missed? Arch Dis Child 102:376-379, 2017.

Wigal SB, Childress AC, Belden HW, Berry SA: NWP06, an extended-release oral suspension of methylphenidate, improved attention-deficity/hyperactivity disorder symptoms compared with placebo in a laboratory classroom study. J Child Adolesc Psychopharmacol 23:3-10, 2013.

Wigal SB, Kollins SH, Childress AC, Adeyi B: Efficacy and tolerability of lisdexamfetamine dimesylate in children with attentiondeficit/hyperactivity disorder: Sex and age effects and effect size across the day. Child Adolesc Psychiatry Ment Health 4:32, 2010.

Wigal SB, Kollins SH, Childress AC, Squires L: A 13-hour laboratory school study of lisdexamfetamine dimesylate in school-aged children with attention-deficit/hyperactivity disorder. Child Adolesc Psychiatry Ment Health 3;17, 2009.

Yamashita H: Prevalance of ADHD. Japanese J Clin Psychopharmacol 8:871-874, 2005 [Japanese].

Zimovetz EA, Beard SM, Hodgkins P, Bischof M, Mauskopf JA, Setyawan J: A cost-utility analysis of lisdexamfetamine versus atomoxetine in the treatment of children and adolescents with attention-deficit/hyperactivity disorder and inadequate response to methylphenidate. CNS Drugs 30:985-996, 2016.

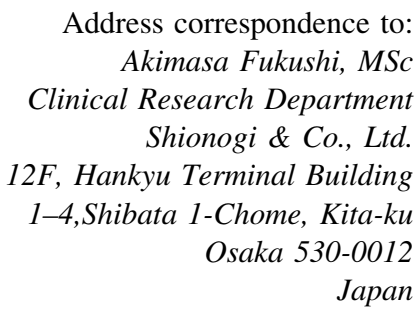

E-mail: akimasa.fukushi@shionogi.co.jp 